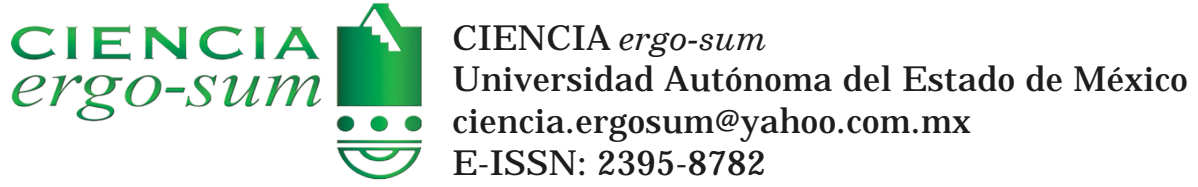

\title{
Prácticas de conservación de suelo para desarrollar resiliencia ante el cambio climático en Santa Cruz Moxolahuac, Puebla, México
}

Yescas Sánchez, Roberto; Cabañas Zárate, Alma Elisa

Prácticas de conservación de suelo para desarrollar resiliencia ante el cambio climático en Santa Cruz Moxolahuac, Puebla, México

CIENCIA ergo-sum, vol. 28, núm. 1, marzo-junio 2021 | e110

Universidad Autónoma del Estado de México, México

Esta obra está bajo una Licencia Creative Commons Atribución-NoComercial-SinDerivar 4.0 Internacional.

Yescas Sánchez, R. y Cabañas Zárate, A. E . (2021). Prácticas de conservación de suelo para desarrollar resiliencia ante el cambio climático en Santa Cruz Moxolahuac, Puebla, México. CIENCIA ergo-sum, 28(1). https://doi. org/10.30878/ces.v28n1a4 


\title{
Prácticas de conservación de suelo para desarrollar resiliencia ante el cambio climático en Santa Cruz Moxolahuac, Puebla, México
}

\section{Soil conservation practices to develop resilient in response to climate change at Santa Cruz Moxolahuac, Puebla, Mexico}

\author{
Roberto Yescas Sánchez \\ Universidad Popular Autónoma del Estado de Puebla, México \\ roberto.yescas@hotmail.com \\ (D) https://orcid.org/0000-0002-9943-2182 \\ Alma Elisa Cabañas Zárate \\ Universidad Popular Autónoma del Estado de Puebla, México \\ almaelisacz@gmail.com \\ (10) https://orcid.org/0000-0002-1119-378X
}

\section{Resumen}

Se describen las prácticas de conservación de suelo realizadas por nueve agricultores de Santa Cruz Moxolahuac, Puebla, México, a partir del modelo teórico transaccional (Holahan, 2006). Se aplicaron dos técnicas de investigación: observación sistematizada para medir los componentes ambiental y conductual, y encuesta para medir el componente psicológico. Los resultados son clasificados en tres tipos de incongruencias, las cuales provocan que no se alcance la resiliencia en los sistemas agrícolas. Por ello, la propuesta de intervención socioambiental sería lograr la correspondencia entre las necesidades ambientales que presenta el sistema agrícola y los conocimientos, las actitudes y los comportamientos cotidianos del agricultor respecto a sus prácticas de conservación de suelo.

Palabras CLAVE: conservación de suelo, resiliencia, sistemas agrícolas, cambio climático.

\begin{abstract}
This research focuses on describing the soil conservation practices of the nine farmers from Santa Cruz Moxolahuac, Puebla, Mexico. The transactional model theory (Holahan, 2006) is used to describe these practices. Two research techniques are used: systematic observation is used to measure both environmental and behavioral components, and a survey to measure the psychological component. The results are classified into three kinds of inconsistencies, which do not allow resilience to develop in the farming systems. Thus, the social - environmental intervention proposal for this investigation is to reach a coherent connection between the environmental needs of the farming systems and the farmer's knowledge, attitudes and behaviors regarding their soil conservation practices.
\end{abstract}

KEYWORDS: soil conservation, resilience, farming systems, climate change.

\section{INTRODUCCIÓN}

En la actualidad el cambio climático es una amenaza para la producción agrícola, principalmente en comunidades rurales pobres con climas que van desde áridos a húmedos (Altieri y Nicholls, 2008). Este tipo de comunidades son las más vulnerables debido a sus condiciones de pobreza que al combinarse con tormentas intensas o sequías afectan tanto la producción agrícola como el sustento de las familias (Purnamita y Morton, 2014), ya que, por un lado, los agricultores suelen carecer de los conocimientos y recursos necesarios para enfrentar las consecuencias de un evento climático extremo y, por otro, en los sistemas agrícolas es común que no se cuente con las condiciones ambientales necesarias para su recuperación inmediata. 
Santa Cruz Moxolahuac es una comunidad rural potencialmente vulnerable ante el cambio climático por dos situaciones. En primer lugar, al tener un clima templado húmedo (INEGI, 2015), es propensa a padecer cambios en la temperatura promedio y al régimen de lluvias (IPCC, 2014). En segundo lugar, sus habitantes carecen de los recursos económicos y tecnológicos para enfrentar las consecuencias de dichos cambios.

Como antecedente, los fenómenos climáticos que más han afectado los cultivos de Moxolahuac son el exceso de lluvia y los fuertes vientos. Las consecuencias han sido la alteración directa en la cantidad de producción, ya que la mayoría son cultivos de temporal, y la afectación a la estabilidad económica y alimentaria de los agricultores y sus familia debido a que son cosechas dirigidas al autoconsumo o trueque.

$\mathrm{Al}$ respecto, algunos científicos han realizado investigaciones sobre el cambio climático y su impacto en la agricultura. Sin embargo, se consideran modelos de simulación porque no se contemplan variables específicas de cada lugar, características edafológicas, entre otras (Granados y Sarabia, 2013; Ramírez et al., 2014).

Si bien son demasiadas las variables a considerar, el conocimiento y las prácticas agrícolas y de conservación de suelo, agua y bosque del agricultor son relevantes para la intervención socioambiental; lo anterior se comprueba con los resultados logrados por Holt-Giménez (2002) ante los efectos del cambio climático, ya que a pesar de la presencia del huracán Mitch en la comunidad rural de Nicaragua, las parcelas con técnicas agroecológicas presentaron mayor cobertura de suelo y de vegetación, así como menor erosión y pérdidas económicas.

En este sentido, el concepto de resiliencia ha emergido como una de las respuestas para disminuir los efectos del cambio climático en los terrenos de cultivo, puesto que se define como la capacidad de un sistema agrícola de continuar produciendo alimentos a pesar de haber sufrido una perturbación climática extrema (Altieri y Nicholls, 2013). Por lo tanto, algunos investigadores han dedicado sus esfuerzos a determinar qué acciones y condiciones generan resiliencia en dichos sistemas (Altieri, 2002).

Actualmente, se ha comprobado la existencia de ciertas prácticas de conservación de suelo para la generación de resiliencia y, de igual manera, se sabe que ciertas características ambientales de tipo paisajísticas y agroecosistémicas son determinantes para su desarrollo (Holt-Giménez, 2002).

De acuerdo con lo anterior, este artículo busca describir las prácticas de conservación de suelo realizadas por algunos agricultores de la comunidad Moxolahuac a partir de los conocimientos, las actitudes y los comportamientos que tienen para su uso en relación con las condiciones ambientales que presentan sus sistemas agrícolas, con el fin de conocer la capacidad que tienen estos últimos para la resiliencia.

El modelo teórico con el cual se realizó esta investigación fue la perspectiva transaccional (citado en Holahan, 2006), que es adaptado del trabajo de Albert Bandura en 1978 y respaldado también por los principios de Kurt Lewin. Este modelo se caracteriza por definir tres componentes para explicar la conducta humana: ambiental, psicológico y conductual, los cuales se influyen recíprocamente de forma directa o indirecta, y cada variable incluida en los componentes puede funcionar como variable independiente, mediadora o dependiente, según el problema particular presentado y el enfoque analítico que se elija (Bandura, 1978). Bajo esta perspectiva, el comportamiento humano sería el resultado de la adaptación que tiene la persona ante la interacción de los elementos que conforman estos tres componentes en su vida cotidiana.

Para los fines de este artículo, el componente psicológico fue definido por los conocimientos y las actitudes que presentaban los agricultores respecto al tema de conservación del suelo y de cuatro prácticas de conservación; en cuanto al componente conductual se consideró la aplicación de dichas prácticas en su terreno de cultivo. Adicionalmente, el componente ambiental se delimitó por la medición de las condiciones paisajísticas y agroecológicas que presentaban los sistemas agrícolas de cada agricultor.

Desde un enfoque cognoscitivista, el conocimiento es el producto de un proceso llamado aprendizaje, y "el aprendizaje se entiende como una actividad mental mediante la cual se alcanza la estructuración interna del conocimiento" (Estévez, 2002: 51). Este producto se encuentra compuesto por proposiciones, imágenes, conceptos y esquemas (Gagné, Yekovich y Yekovich, 1993), los cuales van cambiando a lo largo de la vida, ya sea porque se adicionan nuevos o se reestructuran. 
Cuando un conocimiento sobre un tema es el resultado de la combinación de varias proposiciones, imágenes y conceptos, se está hablando del término esquema. Los esquemas se pueden clasificar en una lógica del más básico al más complejo según los alcances que tiene cada uno de ellos de la siguiente forma (Estévez, 2002): a) el declarativo, el cual se refiere al qué de las cosas; $b$ ) el procedimental, a los conocimientos sobre cómo hacer algo; $c$ ) el situacional o contextual, a los conocimientos del cuándo se requiere hacer uso del conocimiento declarativo o procedimental. Al respecto, una persona que posea nada más conocimiento declarativo difícilmente podrá actuar de manera efectiva en su ambiente, ya que desconoce el cómo, cuándo y para qué hacerlo; aunado a eso, su actuar se verá limitado si desconoce las características de su ambiente físico y social. Por lo tanto, el conocimiento y el comportamiento humano se encuentran vinculados (Bransford, Brown y Cocling, 2000).

En cuanto a las actitudes, éstas fueron consideradas con el objetivo de conocer la tendencia a favor o en contra del tema de conservación de suelo y sus correspondientes prácticas para explicar el comportamiento humano resultante de los participantes y las posibilidades de resistencia ante un posible cambio en la conducta.

Por otro lado, los componentes conductual y ambiental del modelo transaccional se definieron a través de variables propuestas por las referencias seleccionadas. En cuanto al componente conductual, se observó si el agricultor aplica alguna práctica de conservación de suelo con base en lo esperado desde el punto de vista técnico. Hablar de este tipo de prácticas refiere a la aplicación de procedimientos que contribuyan a conservar las características físicas, químicas y microbiológicas del suelo con la intención de mantener su capacidad productiva. En suma, pueden reducir o eliminar el arrastre o la pérdida del suelo causada por la acción de la lluvia y el viento y, de igual modo, mantener o aumentar su fertilidad y lograr mayor productividad en los cultivos (FHIA, 2011). Con base en las características de los terrenos de cultivo estudiados, y considerando la forma de aumentar su resiliencia, se tomaron en cuenta cuatro prácticas de conservación de suelo: $a$ ) barrera viva, $b$ ) cobertura de suelo, $c$ ) terraza y d) drenaje agrícola.

En cuanto al componente ambiental, las variables involucradas se definieron bajo los conceptos nivel depaisaje y nivel de agroecosistema.

El nivel de paisaje está integrado por tres variables:

a) Ecología del paisaje: es el estudio de la composición, estructura y función de los paisajes; la importancia que tiene se basa en su incidencia sobre la biodiversidad (Nogués y Pérez, 2001). En cuanto a los alcances de este artículo, se considera relevante definir la composición del paisaje solamente, el cual es un indicador del número y cantidad de diferentes hábitats en un área dada. Este indicador tiene una fuerte correlación con la biodiversidad, ya que entre más heterogénea sea un área, mayor probabilidad de diversidad de especies tendrá y, por lo tanto, mayores probabilidades de sustentabilidad y resiliencia (Fisher, Boucher y Attwood, 2013).

b) Pendiente: la medición de la pendiente es trascendental, pues los terrenos que se encuentran en zonas inclinadas corren mayores riesgos en cuanto a pérdidas en los cultivos. Adicionalmente, un terreno inclinado es susceptible a la pérdida de suelo y nutrientes por la escorrentía de aguas pluviales (Altieri y Nicholls, 2013; Henao, 2013, Holt-Giménez, 2002).

c) Cercanía a bosques: contar con un bosque cercano es un apoyo, dado que brinda varios servicios ambientales como captación y filtración de agua, generación de oxígeno, protección de la biodiversidad, retención del suelo, entre otros (CONAFOR, 2015).

Por otra parte, respecto a las variables del nivel de agroecosistema se consideraron las siguientes:

a) Estratificación vegetal: se entiende como la clasificación de las plantas de una determinada región en estratos. La presencia de una alta estratificación puede disminuir la erosión por viento y agua, así como evitar la pérdida de suelo y ayudar en la formación de suelo orgánico (Altieri y Nicholls, 2013). 
b) Permeabilidad: con el fin de conocer el aprovechamiento del agua sobre los cultivos, se realizaron las mediciones que se presentan a continuación.

- Permeabilidad del suelo: es la propiedad con la que el agua pasa a través de los poros. Depende de varios factores como el tamaño y la forma de las partículas que componen el suelo, la forma y el arreglo de los poros, entre otros. Se dice que un material es permeable cuando contiene vacíos continuos, los cuales existen en todos los suelos, incluyendo las arcillas más compactas (Angelone, Garibay y Cauhapé, 2006). Como la permeabilidad depende de las características del suelo, se seleccionaron dos mediciones más: análisis granulométrico y análisis de humedad.

- Granulometría: se refiere a la repartición de las partículas sólidas del suelo en tamaño de partículas. Como el rango de las partículas es continuo, se ha subdividido en diferentes clases de tamaño. La subdivisión más común es en rangos de suelo que van de 0 a 2 mm (Moeys, 2015). A partir de los porcentajes de cada tamaño de partícula, se puede determinar la clase textural del suelo: arena, arcilla y limo (Whiting, Wilson y Card, 2002).

- Humedad del suelo: se refiere a la presencia de agua en el suelo. De manera que donde se encuentre saturado es probable que el agua permee y filtre en menor cantidad (Angelone, Garibay y Cauhapé, 2006).

\section{Metodología}

\section{1. Tipo de estudio}

La investigación se desarrolló con base en el enfoque cuantitativo de tipo no experimental (Hernández, Fernández y Baptista, 2014; Gerring, 2012), el cual consiste en observar sistemáticamente situaciones o fenómenos existentes a través de los hechos o factos. Aunado a esto, también se podría considerar como una investigación transversal con alcance descriptivo, ya que con los resultados se podrán conocer las prácticas de conservación de suelo que llevaron a cabo los participantes del estudio durante el segundo semestre de 2015 considerando las condiciones ambientales de sus terrenos de cultivo.

Se emplearon dos técnicas de estudio: a) observación sistematizada a través de la tabula de observación y $b$ ) la encuesta, la cual se concretó con un cuestionario.

\subsection{Participantes}

La determinación de los participantes se realizó a partir de una muestra no probabilística del tipo bola de nieve y por conveniencia (Rubio y Varas, 2004). La justificación para elegirlos por medio del método de bola de nieve fue debido a la falta de interés por parte de los agricultores en participar; por ello, se le solicitó al primer contacto hacer la recomendación con el siguiente. Asimismo, la selección de los sujetos de estudio por el método por conveniencia fue para lograr heterogeneidad geográfica en los sistemas agrícolas posibles de ser estudiados, es decir, se seleccionaron a los agricultores interesados cuyos terrenos de cultivo estuvieran distribuidos geográficamente alrededor del territorio que conforma Santa Cruz Moxolahuac, y de este modo tener resultados de distintos lugares de la comunidad.

Se seleccionaron nueve participantes en total, quienes se clasificaron en dos grupos: ejidatarios y propietarios. Los ejidatarios son el conjunto de personas a quienes se les otorgó uno o varios terrenos para cultivar por parte del gobierno y los propietarios son los poseedores del terreno que cultivan. En concreto, cuatro de los nueve agricultores fueron ejidatarios y el resto, propietarios. Otras características de los participantes son las siguientes: su 
edad promedio es de 50 años, todos del sexo masculino, llevan en promedio 30 años cultivando, cinco de nueve tienen hasta la mitad de los estudios de nivel primaria, dos han terminado la secundaria y el resto cuenta con la preparatoria.

En el mapa 1 se puede observar el espacio que conforma el territorio de Santa Cruz Moxolahuac: en el centro se encuentran los asentamientos humanos y a su alrededor se ubican los nueve casos considerados.

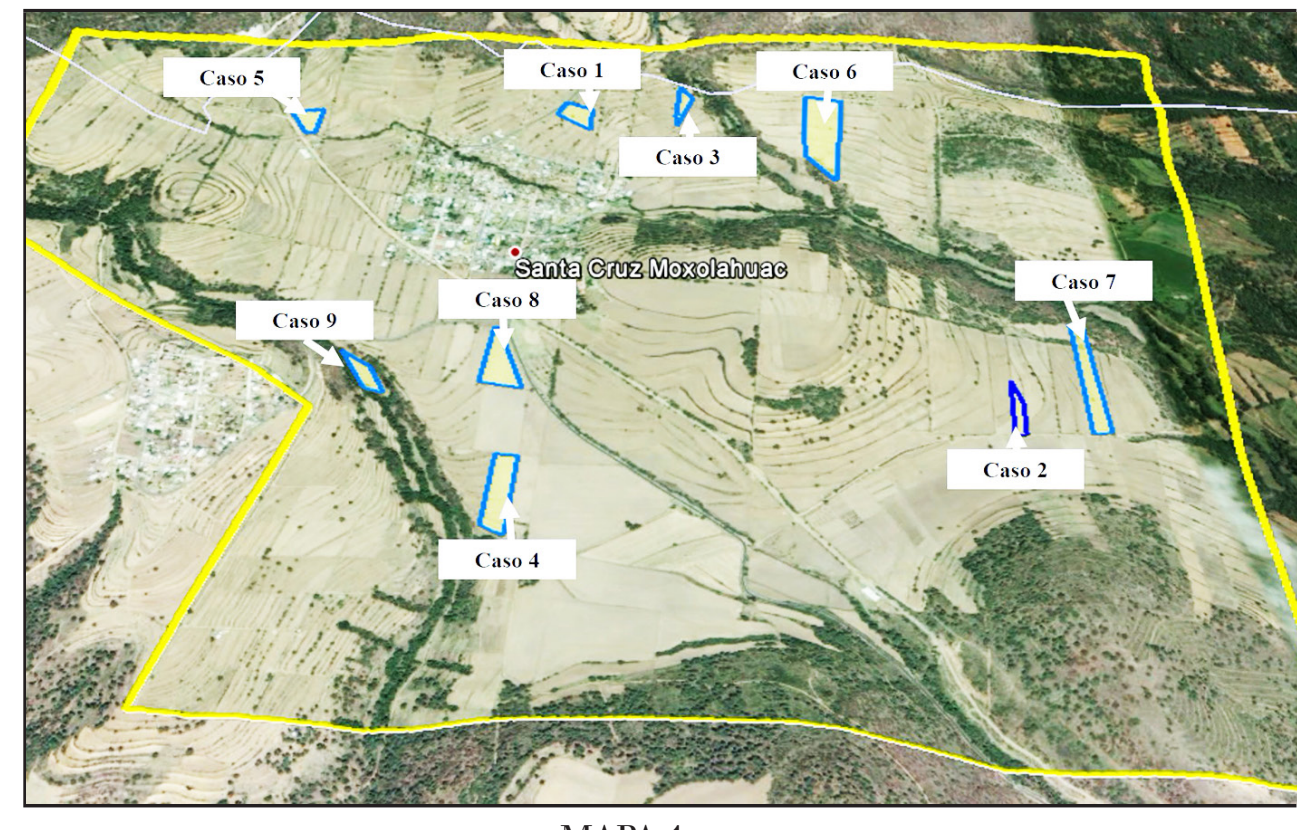

MAPA 1

Delimitación del territorio de Santa Cruz Moxolahuac y los nueve casos de estudio Fuente: elaboración propia.

\subsection{Instrumentos}

Por un lado, se utilizó la observación sistematizada, la cual se caracteriza por tener delimitados tanto el campo que se va a estudiar (lugares y participantes) como los aspectos concretos o conductas sobre las que se va a centrar la atención (Rubio y Varas, 2004). Dicha observación se realizó por medio de una tabula en la que se registraron todas las mediciones del componente ambiental como las correspondientes del componente conductual.

Por otro lado, se consideró el uso de la técnica llamada encuesta, la cual se concretó a través del diseño y la aplicación de un cuestionario a los participantes de forma personal. La información obtenida con este instrumento fue sobre sus características personales y los conocimientos que tienen (declarativo, procedimental o situacional) acerca del tema de conservación de suelo y de las cuatro prácticas de conservación seleccionadas, así como la actitud positiva que poseen sobre ellas.

\subsection{Procedimiento}

A continuación se presentan los pasos metodológicos:

1. Debido a la escasez de datos estadísticos y geográficos del lugar, primero se delimitó la zona de estudio. Esto es, por medio del registro de coordenadas satelitales, se estableció el polígono del espacio ocupado por la comunidad de Santa Cruz Moxolahuac (mapa 1). 
2. Se realizó la selección de los participantes con la muestra no probabilística de tipo bola de nieve y por conveniencia. En el momento del contacto y una vez confirmada la participación del agricultor, se ubicó su sistema agrícola y se agendó una cita con él para la aplicación de los dos instrumentos.

3. Se aplicó la tabula de observación con el fin de dar paso a la definición de las preguntas del cuestionario. Estas preguntas se concretaron según las prácticas de conservación de suelo susceptibles a desarrollar la resiliencia en los terrenos de cultivo (en este caso fueron cuatro).

Las mediciones de los indicadores contemplados en la tabula de observación se llevaron a cabo de la siguiente forma:

a) Ecología del paisaje: se midió la composición del paisaje mediante la metodología propuesta por Fisher, Boucher y Attwood (2013). Para la medición de este indicador, se utilizó una imagen satelital proporcionada por Google Earth y las herramientas de medición de este mismo programa.

b) Pendiente: para la medición de la pendiente se utilizó el clinómetro, que fue construido y utilizado de acuerdo con la guía de la FAO (2017). Adicionalmente, la medición de este indicador se expresó en porcentaje y se calculó dividiendo la altura de la pendiente entre su base y multiplicando el resultado por 100. La forma de valorar los resultados de la pendiente se realizó bajo el sistema de semáforo propuesto por Altieri y Nicholls (2013): rojo para los valores que se encuentren por encima de 30\%, amarillo para los que se localicen entre $25 \%$ y $30 \%$ y verde para los ubicados entre $20 \%$ y $24 \%$.

c) Cercanía a bosques: se tomaron las coordenadas geográficas por medio del GPS (Garmin Etrex) y se utilizó un sistema de información geográfica: Google Earth, a partir de la metodología de Valdez, González y De los Santos (2006) con el fin de medir este indicador

d) Estratificación vegetal: en primer lugar, se determinaron los estratos y los rangos de las alturas de cada uno; para ello, se requirió de una cuerda de $5 \mathrm{~m}$ de largo para delimitar el perímetro del círculo de muestreo y un flexómetro para medir la altura de la planta más alta y la más baja dentro del círculo. En segundo lugar, se realizaron las mediciones correspondientes a partir de la metodología de Rodríguez (2008) para obtener el índice y el valor exponencial de Shannon para cada terreno de cultivo. Este último valor indica el número de estratos equivalentes de la parcela (cuadro 3 ).

e) Permeabilidad: la medición de este indicador se determinó a partir de la prueba Matsuo y así conocer el coeficiente de permeabilidad $(k)$, el cual se define como la velocidad de filtración que tiene el agua a través del suelo. La resultante de cada $k$ de permeabilidad en cada caso es valorada de manera cualitativa con los rangos establecidos por Peck, Hanson y Thornburn (2008) y Lambe y Whittman (2004).

En el caso del análisis granulométrico se obtuvo una muestra del suelo y, a partir de un tratamiento se obtuvo la clasificación textural de cada muestra considerando sus porcentajes de arena y material fino. La ubicación de cada caso se logró mediante la calculadora de textura de suelo de la United States Department of Agriculture (USDA, 2016).

Por su parte, el análisis de humedad se realizó al obtener su porcentaje (w) en el suelo muestreado.

4. El cuestionario se diseñó a partir de los resultados del componente ambiental, y posteriormente, se aplicó a los participantes. Los datos obtenidos fueron de tipo fáctico y discursivo. Los fácticos están relacionados con las características personales de los participantes y los discursivos con las respuestas emitidas sobre las preguntas de nivel de conocimiento o actitudinal. Estos últimos fueron valorados con base en la teoría o información técnica de los temas: conservación de suelo y de las cuatro prácticas de conservación seleccionadas para alcanzar la resiliencia. Se midieron los tres tipos de conocimiento: 
declarativo (D), procedimental (P), situacional o contextual (C) y la postura actitudinal (A). Durante el proceso de indagación se consideraron los signos y significados que tienen los agricultores para referirse a cada una de las prácticas de conservación de suelo. Además, el procesamiento y el análisis de los datos se realizó a partir de una rúbrica. Según la respuesta del participante, ésta se valoró en tres sentidos:

- Un punto: la respuesta era correcta según la teoría o si la tendencia actitudinal era a favor de la conservación del suelo o del uso de alguna práctica.

- Medio punto: la respuesta era parcialmente correcta según la teoría o la tendencia actitudinal no era explícitamente a favor de la conservación del suelo o del uso de alguna práctica.

- Cero puntos: la respuesta era incorrecta o el participante no sabía nada al respecto o cuando la tendencia actitudinal era neutra o en contra de la conservación del suelo o del uso de alguna práctica.

5. Al final, se analizaron los resultados de los dos instrumentos y se realizó una discusión y conclusión del estudio.

\section{Resultados}

A continuación se presentan los resultados obtenidos:

a) Componente ambiental:

- Ecología del paisaje: con base en los resultados, el paisaje de la comunidad se compone por dos tipos de hábitats cuya extensión es de $1.3 \mathrm{~km}^{2}$ para el bosque templado y $0.52 \mathrm{~km}^{2}$ para el pastizal, los cuales representan 28\% del territorio total de la zona de estudio; el resto, se encuentra conformado por zonas agrícolas y urbanizadas (cuadro 1).

- Pendiente: se llevaron a cabo cinco mediciones para obtener la pendiente promedio en cada uno de los casos; el promedio en términos de porcentaje obtenido en cada caso se presenta en el cuadro 2.

\section{CUADRO 1}

Tipos de hábitats en Santa Cruz Moxolahuac

\begin{tabular}{|lc|}
\hline Hábitat & Cobertura promedio respecto al territorio en $\mathrm{km}^{2}$ \\
\hline Bosque templado & 1.3 \\
Pastizal/Pradera & 0.52 \\
\hline
\end{tabular}

Fuente: elaboración propia.

- Cercanía a bosques: en primer lugar, se comprobó la existencia de bosque en la zona de estudio con base en el criterio de la UNFCCC (2014). En segundo lugar, se obtuvo la distancia promedio entre el terreno de cultivo y el bosque más cercano a él. Los resultados presentados en el cuadro 2 muestran que el terreno del caso 9 es el más cercano al bosque con $35 \mathrm{~m}$ y le sigue el caso 3 con $95 \mathrm{~m}$ en promedio; el resto está ubicado a una distancia promedio mayor a los $110 \mathrm{~m}$.

- Estratificación vegetal: los resultados muestran al caso 1 con el mayor número de estratos (tres), y al caso 2 y 8 con el menor número (uno) (cuadro 3). 
CUADRO 2

Pendiente y cercanía a bosques por cada caso

\begin{tabular}{|lcc|}
\hline Número de caso & \multicolumn{2}{c|}{ Indicadores } \\
\cline { 2 - 3 } & Pendiente & Cercanía a bosques \\
\cline { 2 - 3 } & Promedio (\%) & Distancia promedio (m) \\
\hline Caso 1 & 15.8 & 170 \\
Caso 2 & 12.4 & 310 \\
Caso 3 & 15.8 & 95 \\
Caso 4 & 12 & 120 \\
Caso 5 & 21.5 & 290 \\
Caso 6 & 17.2 & 140 \\
Caso 7 & 4 & 210 \\
Caso 8 & 5 & 315 \\
Caso 9 & 10.2 & 35 \\
Promedio & 12.65 & 187 \\
\hline
\end{tabular}

Fuente: elaboración propia.

- Permeabilidad: los resultados de esta variable se dividen en tres.

-Permeabilidad del suelo: con base en los resultados, se puede decir que el suelo alcanza a ser permeable; en otras palabras, es posible la filtración del agua en un rango cualitativo de medio a bajo. $\mathrm{Al}$ respecto, sobrepasan dicho rango los casos 3 y 9 (cuadro 3 ).

- Análisis granulométrico: los casos seleccionados fueron el 1, 2, 4, 5 y 9 con la intención de conocer de forma distributiva el tipo de suelo predominante. El análisis granulométrico se realiza a partir de rangos de suelo que van de 0 a $2 \mathrm{~mm}$ (cuadro 3 ).

El tipo de suelo predominante es la arcilla y limo. Según Porta, López-Acevedo y Poch (2014), si la fracción predominante es arcilla, el suelo tendrá las siguientes características: fertilidad química alta (según mineralogía), dificultad de laboreo debido a su elevada plasticidad (estado húmedo) o compacidad (en seco), retención de humedad alta, dificultad en la penetración de raíces, entre otras.

-Análisis de humedad en el suelo: en el cuadro 3 se presentan los porcentajes de humedad en el suelo para cada caso. Los resultados muestran una correlación negativa de 0.75 omitiendo los casos anómalos 2 y 7; es decir, a mayor humedad menor permeabilidad y viceversa.

\section{b) Componente conductual:}

A partir de la observación directa en cada terreno de cultivo, se identificaron las prácticas de conservación de suelo llevadas a cabo por cada agricultor. Como se puede observar en la tabla 1, cobertura de suelo y terraza fueron las dos prácticas de conservación de suelo con mayor frecuencia de aparición de las cuatro existentes en los sistemas agrícolas estudiados. Dichas prácticas se presentan en los casos 1, 2, 3, 4, 6, 7, 8 y 9; sin embargo, en cuanto al caso 5, no se presenta alguna de estas prácticas.

Aunado a lo anterior, considerando los resultados del componente ambiental se pueden deducir las prácticas de conservación de suelo necesarias para lograr la resiliencia en cada uno de los nueve casos. Al respecto, en la tabla 1 se puede observar que no existe congruencia entre la práctica de conservación llevada a cabo por el agricultor y la que en realidad necesita el terreno de cultivo. Por ello, se puede mencionar 
lo siguiente: para el caso 1 y 3 se requiere aplicar solamente barreras vivas, pero estos agricultores aplican terrazas; para el caso 2, 4, 7 y 8 se necesita aplicar tanto barreras vivas como drenajes, pero los casos 2,7 y 8 aplican cobertura de suelo y el caso 4, terrazas; para el caso 5 se requiere de barreras vivas y terrazas, pero este agricultor no aplica alguna práctica y, por último, para los casos 6 y 9 no se necesita alguna práctica de conservación de suelo.

\section{CUADRO 3}

Estratificación vegetal, permeabilidad del suelo, análisis granulométrico y análisis de humedad por cada caso

\begin{tabular}{|c|c|c|c|c|c|c|c|c|c|c|}
\hline \multirow[t]{3}{*}{$\begin{array}{l}\text { Número } \\
\text { de caso }\end{array}$} & \multicolumn{2}{|c|}{$\begin{array}{c}\text { Estratificación } \\
\text { vegetal } \\
\end{array}$} & \multicolumn{3}{|c|}{$\begin{array}{c}\text { Permeabilidad } \\
\text { del suelo }\end{array}$} & \multicolumn{4}{|c|}{$\begin{array}{c}\text { Análisis } \\
\text { granulométrico } \\
\end{array}$} & \multirow{3}{*}{$\begin{array}{c}\text { Análisis de } \\
\text { humedad }\end{array}$} \\
\hline & \multirow[t]{2}{*}{ Índice } & \multirow[t]{2}{*}{$\begin{array}{c}\text { Valor } \\
\text { exponencial }\end{array}$} & \multirow[t]{2}{*}{$\begin{array}{l}\text { Valor } k \text { en } \\
\mathrm{cm} / \mathrm{s}\end{array}$} & \multirow{2}{*}{$\begin{array}{c}\text { Peck, } \\
\text { Hanson y } \\
\text { Thornburn }\end{array}$} & \multirow[t]{2}{*}{$\begin{array}{l}\text { Lambe y } \\
\text { Whittman }\end{array}$} & \multicolumn{4}{|c|}{$\begin{array}{l}\text { Porcentaje de suelo que pasa por la malla } \\
\text { según su tamaño }\end{array}$} & \\
\hline & & & & & & $2 \mathrm{~mm}$ & $1 \mathrm{~mm}$ & $0.25 \mathrm{~mm}$ & $0.075 \mathrm{~mm}$ & \\
\hline Caso 1 & 1.076 & 3.023 & $4.1 \times 10^{-3}$ & Bueno & Media a baja & $92.80 \%$ & $92.14 \%$ & $89.01 \%$ & $63.30 \%$ & 23.462 \\
\hline Caso 2 & 0.374 & 1.501 & $1.3 \times 10^{-3}$ & Bueno & Media a baja & $97.28 \%$ & $96.56 \%$ & $90.00 \%$ & $52.98 \%$ & 18.057 \\
\hline Caso 3 & 0.747 & 2.253 & $1.7 \times 10^{-2}$ & Bueno & Media & - & - & - & - & 16.204 \\
\hline Caso 4 & 0.668 & 2.263 & $2.4 \times 10^{-3}$ & Bueno & Media a baja & $98.44 \%$ & $98.12 \%$ & $91.75 \%$ & $59.17 \%$ & 27.139 \\
\hline Caso 5 & 0.677 & 2.156 & $5.3 \times 10^{-3}$ & Bueno & Media a baja & $98.01 \%$ & $97.24 \%$ & $88.79 \%$ & $61.78 \%$ & 19.642 \\
\hline Caso 6 & 1.008 & 2.807 & $1.05 \times 10^{-3}$ & Bueno & Media a baja & - & - & - & - & 28.445 \\
\hline Caso 7 & 0.855 & 2.775 & $1.8 \times 10^{-3}$ & Bueno & Media a baja & - & - & - & - & 9.241 \\
\hline Caso 8 & 0.058 & 1.079 & $1.6 \times 10^{-3}$ & Bueno & Media a baja & - & - & - & - & 29.266 \\
\hline Caso 9 & 0.950 & 2.586 & $1.5 \times 10^{-2}$ & Bueno & Media & $99.12 \%$ & $98.65 \%$ & 89.77 & $58.21 \%$ & 16.692 \\
\hline Promedio & --- & 2.271 & $4.5 \times 10^{-3}$ & Bueno & Media a baja & - & - & - & - & - \\
\hline
\end{tabular}

Fuente: elaboración propia.

TABLA 1

Prácticas de conservación presentes y requisitos para alcanzar la resiliencia por cada caso

\begin{tabular}{|c|c|c|}
\hline Número del caso & Práctica de conservación de suelo presente & Requisitos para alcanzar la resiliencia \\
\hline Caso 1 & - Terrazas & - Barreras vivas \\
\hline Caso 2 & - Cobertura de suelo & $\begin{array}{l}\text { - Barreras vivas } \\
\text { - Drenajes }\end{array}$ \\
\hline Caso 3 & - Terrazas & - Barreras vivas \\
\hline Caso 4 & - Terrazas & $\begin{array}{l}\text { - Barreras vivas } \\
\text { - Drenajes }\end{array}$ \\
\hline Caso 5 & - Ninguna & $\begin{array}{l}\text { - Barreras vivas } \\
\text { - Terrazas }\end{array}$ \\
\hline Caso 6 & $\begin{array}{l}\text { - Cobertura de suelo } \\
\text { - Barreras vivas }\end{array}$ & • Ninguno \\
\hline Caso 7 & - Cobertura de suelo & $\begin{array}{l}\text { - Barreras vivas } \\
\text { - Drenajes }\end{array}$ \\
\hline Caso 8 & - Cobertura de suelo & $\begin{array}{l}\text { - Barreras vivas } \\
\text { - Drenajes }\end{array}$ \\
\hline Caso 9 & - Terrazas & - Ninguno \\
\hline
\end{tabular}

c) Componente psicológico:

En cuadro 4 se observan los resultados. Por un lado, en una lectura horizontal, de izquierda a derecha, se puede conocer el puntaje total por cada uno de los agricultores. Por otro lado, en una lectura vertical, de arriba hacia abajo, se puede conocer el puntaje total sobre un nivel de conocimiento o actitudinal de la práctica correspondiente. 
Considerando cada uno de los casos, los que tienen mayor puntaje son el caso 1,4 y 8 , donde destaca este último por obtener 13.5 puntos. Lo anterior significa que estos agricultores tienen mayores conocimientos correctos y una tendencia actitudinal positiva hacia la conservación de suelo y ante las cuatro prácticas estudiadas, a diferencia del resto de los agricultores seleccionados.

Por otra parte, el caso 6 es el que tiene el menor puntaje (2). Esto quiere decir que es el agricultor con los menores conocimientos correctos y una tendencia actitudinal neutra o negativa hacia la conservación del suelo y a las cuatro prácticas consideradas en este artículo.

El resto de los casos tiene puntajes intermedios, lo que significa que tienen conocimientos y actitudes respecto a la conservación de suelo y ante las cuatro prácticas mencionadas en un puntaje total que va de 8.5 a 10; es decir, estos casos se acercan más al extremo superior que al inferior en términos de puntaje total obtenido.

\section{CUADRO 4}

Conocimientos y actitudes sobre conservación del suelo y de cuatro prácticas de conservación por cada caso

\begin{tabular}{|c|c|c|c|c|c|c|c|c|c|c|c|c|c|c|c|c|c|c|c|c|c|}
\hline \multirow{3}{*}{ Caso } & \multirow{2}{*}{\multicolumn{4}{|c|}{$\begin{array}{c}\text { Conservación del } \\
\text { suelo }\end{array}$}} & \multicolumn{16}{|c|}{ Prácticas de conservación de suelo } & \multirow{3}{*}{ Total } \\
\hline & & & & & \multicolumn{4}{|c|}{ Barreras vivas } & \multicolumn{4}{|c|}{ Cobertura de suelo } & \multicolumn{4}{|c|}{ Terrazas } & \multicolumn{4}{|c|}{ Drenajes } & \\
\hline & $\mathrm{D}$ & $\mathrm{P}$ & $\mathrm{C}$ & $\mathrm{A}$ & $\mathrm{D}$ & $\mathrm{P}$ & $\mathrm{C}$ & $\mathrm{A}$ & $\mathrm{D}$ & $\mathrm{P}$ & $\mathrm{C}$ & $\mathrm{A}$ & $\mathrm{D}$ & $\mathrm{P}$ & $\mathrm{C}$ & $\mathrm{A}$ & $\mathrm{D}$ & $\mathrm{P}$ & $\mathrm{C}$ & $\mathrm{A}$ & \\
\hline 1 & 1 & 0 & 1 & 0.5 & 0 & 0 & 0 & 0 & 0 & 0 & 0 & 0 & 1 & 1 & 1 & 1 & 1 & 1 & 1 & 1 & 10.5 \\
\hline 2 & 0 & 0 & 1 & 1 & 0 & 0 & 0 & 0 & 0 & 0 & 0 & 0 & 1 & 1 & 1 & 1 & 1 & 0 & 1 & 1 & 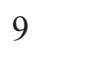 \\
\hline 3 & 0.5 & 0.5 & 1 & 1 & 0 & 0 & 0 & 0 & 1 & 1 & 0.5 & 0.5 & 0.5 & 0 & 1 & 1 & 0 & 0 & 0 & 0 & 8.5 \\
\hline 4 & 1 & 1 & 1 & 1 & 0 & 0 & 0 & 0 & 0 & 0 & 0 & 0 & 0.5 & 1 & 0.5 & 0.5 & 1 & 1 & 1 & 1 & 10.5 \\
\hline - & 0.5 & 1 & 0.5 & 0.5 & 0 & 0 & 0 & 0 & 0 & 0 & 0 & 0 & 0.5 & 1 & 0.5 & 0.5 & 1 & 1 & 1 & 1 & 9 \\
\hline 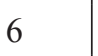 & 0 & 1 & 0.5 & 0.5 & 0 & 0 & 0 & 0 & 0 & 0 & 0 & 0 & 0 & 0 & 0 & 0 & 0 & 0 & 0 & 0 & 2 \\
\hline 7 & 1 & 1 & 0.5 & 0.5 & 0 & 0 & 0 & 0 & 0 & 0 & 0 & 0 & 0.5 & 1 & 0.5 & 0.5 & 0.5 & 1 & 1 & 1 & 9 \\
\hline 0 & 0.5 & 1 & 1 & 1 & 0 & 0 & 0 & 0 & 0 & 0.5 & 1 & 1 & 0.5 & 1 & 1 & 1 & 1 & 1 & 1 & 1 & 13.5 \\
\hline , & 0 & 1 & 1 & 1 & 0 & 0 & 0.5 & 0.5 & 0 & 0 & 0 & 0 & 0.5 & 1 & 1 & 1 & 1 & 0.5 & 0.5 & 0.5 & 10 \\
\hline Total & 4.5 & 6.5 & 7.5 & 7 & 0 & 0 & 0.5 & 0.5 & 1 & 1.5 & 1.5 & 1.5 & 5 & 7 & 6.5 & 6.5 & 6.5 & 5.5 & 6.5 & 6.5 & 82 \\
\hline
\end{tabular}

Fuente: elaboración propia.

Nota: $\mathrm{D}=$ conocimiento declarativo, $\mathrm{P}=$ conocimiento procedimental, $\mathrm{C}=$ conocimiento situacional o contextual y $\mathrm{A}=$ actitud.

\section{Discusión}

El modelo teórico transaccional (citado en Holahan, 2006) propone la explicación de la conducta humana a partir del estudio de tres componentes: ambiental, psicológico y conductual. Dicho modelo parte de la idea de considerar al comportamiento humano cotidiano como el resultado de la intersección entre estos tres componentes; es decir, es el comportamiento el resultado de un proceso de adaptación continuo donde el ambiente y la persona se determinan recíprocamente.

En este trabajo se delimitó el estudio a un comportamiento particular: las prácticas de conservación de suelo de nueve agricultores y, con base en los resultados, se descubrió que existen incongruencias entre lo que necesita el suelo para ser resiliente ante el cambio climático (la parte ambiental), el conocimiento y las actitudes que tienen los agricultores al respecto (la parte psicológica) y lo que hacen finalmente ellos a la hora de cultivar (la parte conductual). Dichas incongruencias no permiten establecer la resiliencia en los sistemas agrícolas estudiados, y cabe la posibilidad de que no se logre en el futuro si las condiciones se mantienen como están hasta el momento.

En este sentido, los resultados alcanzados permiten entender lo dicho por Purnamita y Monton (2014) referente a la vulnerabilidad de las comunidades ante el cambio climático, ya que esto se debe principalmente a las condiciones de pobreza de los agricultores combinado con la falta de conocimiento y de recursos por parte de ellos para enfrentar las consecuencias de un evento climático extremo. 
No obstante, existe la posibilidad de desarrollar resiliencia en los sistemas agrícolas estudiados, ya que con base en los resultados obtenidos se podría pensar en acciones adaptadas a las condiciones sociales, económicas y ambientales de cada caso revisado; un ejemplo al respecto es el de Holt-Giménez (2002).

Adicionalmente, se puede decir que mientras los tres componentes estudiados interactúen de forma congruente, los nueve agricultores podrán enfrentar de manera económica y eficiente las consecuencias del cambio climático a la hora de cosechar y cultivar.

Por lo tanto, este trabajo se suma a los esfuerzos que se vienen dando respecto al aumento de la resiliencia en los sistemas agrícolas en general (Altieri, 2002) y, en particular, da un paso al conocimiento y a la descripción de lo que puede estar ocurriendo en esta comunidad dejando a un lado las investigaciones en donde se elaboran propuestas a partir de casos simulados (Granados y Sarabia, 2013; Ramírez et al., 2014).

\section{Conclusiones y Prospectiva}

La única característica ambiental presentada que favorece la resiliencia en los terrenos de cultivo estudiados es la pendiente, ya que resulta ser baja porque no alcanza a sobrepasar el $20 \%$ de inclinación. En contraste, el resto de las características ambientales no favorece la resiliencia en la zona de estudio. Un característica especial es el de permeabilidad, pues existe un alto nivel que puede favorecer la resiliencia, pero debido a los resultados del análisis granulométrico y los de humedad se puede determinar que las condiciones del suelo no son favorables para la resiliencia porque se encontró predominantemente un suelo de tipo limo y arcilla y exceso de humedad, lo cual afecta la permeabilidad del agua, sobre todo en temporadas de lluvia.

Por lo anterior, los agricultores en su mayoría necesitan aplicar las prácticas barrera viva y drenaje agrícola con el fin de desarrollar la resiliencia en sus terrenos de cultivo.

Asimismo, los nueve participantes presentan conocimiento correcto y actitud positiva en tres de los cinco temas estudiados. En primer lugar se encuentra el tema de conservación de suelo con un puntaje total de 25.5, y en segundo lugar, existe un empate entre el tema de terraza y drenaje agrícola con un puntaje total de 25 en cada uno. En cuanto a los temas con menor puntaje total, destaca cobertura de suelo con 5.5, y le sigue, barrera viva con un punto. Al respecto, se puede decir que los agricultores se encuentran sensibilizados en la conservación del suelo y, asimismo, tienen los conocimientos básicos para realizar acciones que les permitan lograrlo. Sin embargo, esto no se traduce en el hecho de que ellos puedan desarrollar la resiliencia en sus sistemas agrícolas, ya que tendrían que aplicar alguna de las cuatro prácticas de conservación de suelo propuestas. En cuanto a terraza y drenaje agrícola, ambas tienen un alto puntaje tanto de conocimientos correctos como de nivel actitudinal positivo para su uso, pero en cuanto a cobertura de suelo y barreras vivas ocurre lo contrario.

Considerando los resultados de los tres componentes, y una vez analizado cada uno de ellos, es posible concluir lo siguiente: ocho de nueve casos estudiados realizan incongruentemente prácticas de conservación de suelo, las cuales no permiten generar resiliencia en sus sistemas agrícolas. Las incongruencias se manifiestan de tres formas:

a) Incongruencia de tipo ambiental: existe correspondencia entre el componente psicológico y conductual, pero no con el ambiental. En otras palabras, el agricultor puede conocer y poseer una actitud a favor de una práctica de conservación del suelo y, por lo tanto, la lleva acabo en su sistema agrícola; no obstante, desconoce las necesidades específicas de su terreno, las cuales requieren de la aplicación de otras prácticas, o no necesariamente de las que actualmente lleva a cabo. Lo anterior aplica para el caso 1.

b) Incongruencia de tipo conductual: existe correspondencia entre el componente psicológico y el ambiental, pero no con el conductual. En este sentido, el agricultor tiene conocimientos y una actitud positiva para llevar a cabo ciertas prácticas de conservación de suelo; sin embargo, no las aplica en su sistema agrícola a pesar de ser requeridas. Quizá una forma de entender esta situación sea que los participantes no tengan 
los recursos económicos o materiales para llevar a cabo las prácticas que conocen o tal vez que, a pesar de tener los conocimientos y la actitud positiva, desconocen las necesidades de su terreno para aumentar la resiliencia. Esta incongruencia se observa en los casos 2, 4, 5, 7 y 8.

c) Incongruencia de tipo psicológica: no se presenta correspondencia entre el componente psicológico y el conductual. Es decir, los participantes llevan a cabo ciertas prácticas de conservación de suelo sin poseer los conocimientos o las actitudes necesarias para ello. A esto se le ha llamado una conducta imitativa (Bandura, 1962), ya que los participantes mencionaron durante la aplicación del cuestionario que realizan ciertas prácticas en su terreno basadas en lo que los demás agricultores hacen sin conocer qué, cómo, para qué o cuándo realizarlas. Este tipo de incongruencia aplica para los casos: 2, 3, 4, 6, 7 y 8.

El caso 9 es excepcional debido a la congruencia existente entre los tres componentes. En otras palabras, este agricultor aplica terrazas, tiene los conocimientos y la actitud positiva para llevar a cabo dicha práctica y, por último, por las características que presenta su sistema agrícola solamente se necesita que siga aplicando dicha práctica.

Finalmente, los descubrimientos del artículo resaltan la importancia de la congruencia entre los tres componentes del modelo transaccional para actuar de manera efectiva en el ambiente y, en este caso, en cuanto al desarrollo de la resiliencia en los sistemas agrícolas ante el cambio climático. Por ello, la intervención socioambiental que se pudiera implementar en Santa Cruz Moxolahuac sería en tres sentidos:

a) Informar a los agricultores sobre las condiciones ambientales de su sistema agrícola y las prácticas de conservación de suelo que puedan desarrollar la resiliencia.

b) Enseñar a los participantes el qué, cómo, cuándo y para qué de cada una de las prácticas recomendadas.

c) Diseñar estrategias económicas para la implementación de cada práctica de conservación de suelo.

\section{ReFERENCIAS}

Altieri, M. A. (2002). Agroecology: The science of natural resource management for poor farmers in marginal environments. Agriculture, Ecosystems and Environment, 93. Retrieved from http://www.sciencedirect. $\mathrm{com} / \mathrm{science} /$ article/pii/S0167880902000853

Altieri, M. y Nicholls, C. (2008). Los impactos del cambio climático sobre las comunidades campesinas y de agricultores tradicionales y sus respuestas adaptativas. Agroecología, 3. Disponible en http://revistas. um.es/agroecologia/article/view/95471

Altieri, M. A. y Nicholls, C. I. (2013). Agroecología y resiliencia al cambio climático: principios y consideraciones metodológicas. Agroecologia, 8. Disponible en http://revistas.um.es/agroecologia/article/view/182921

Angelone, S., Garibay, M. T. y Cauhapé, M. (2006). Geología y geotecnia:permeabilidad de suelos. Disponible en http://www.fceia.unr.edu.ar/geologiaygeotecnia/Permeabilidad\%20en\%20Suelos.pdf

Bandura, A. (1978). The self system in reciprocal determinism. American Psychologist, 33. Disponible en http:// psycnet.apa.org/psycinfo/1979-08427-001

Bandura, A. (1962). Social learning through imitation. Lincoln: University of Nebraska Press.

Bransford, J., Brown, A. L., \& Cocking, R. T. (2000). How people learn: Brain, mind, experience, and school. Washington, D. C.: National Academy Press.

CONAFOR (Comisión Nacional Forestal). (2015). Servicios ambientales. Disponible en http://www.conafor. gob.mx/web/temas-forestales/servicios-ambientales 
Estévez, E. H. (2002). Enseñar a aprender: estrategias cognitivas. México: Paidós.

Fisher, J. R. B., Boucher, T. M., \& Attwood, S. K. (2013). How do we know an agricultural system is sustainable? Nature. Retrieved from http://www.nature.org/science-in-action/science-features/ag-sustainability-metrics.pdf

FAO (Food and Agriculture Organization). (2017). Medición de ángulos verticales y pendientes. Disponible en $\mathrm{ftp}: / / \mathrm{ftp}$.fao.org/fi/CDrom/FAO_training/FAO_training/general/x6707s/x6707s04.htm\#82a

FHIA (Fundación Hondureña de Investigación Agrícola). (2011). Guía sobre prácticas de conservación de suelos. Disponible en http://www.fhia.org.hn/dowloads/guia_conservacion_de_suelos.pdf

Gagné, E. D., Yekovich, C. W., \& Yekovich, F. R. (1993). The cognitive psychology of school learning (2nd ed.). New York: HarperCollins.

Gerring, J. (2014). Metodología de las ciencias sociales. España: Alianza.

Granados, R. y Sarabia, A. A. (2013). Cambio climático y efectos en la fenología del maíz en el DDR-Toluca. Revista Mexicana de Ciencias Agrícolas, 4. Disponible http://www.scielo.org.mx/scielo.php?pi$\mathrm{d}=$ S200709342013000300008\&script=sci_arttt

Henao, A. (2013). Propuesta metodológica de medición de la resiliencia agroecológica en sistemas socio-ecológicos: un estudio de caso en los Andes colombianos. Agroecología, 8. Disponible en http://revistas.um.es/ agroecologia/article/view/183031

Hernández, R., Fernandez, C. y Baptista, P. (2014). Metodología de la investigación (sexta edición). México: McGraw-Hill.

Holahan, C. J. (2006). Psicología ambiental. México: Limusa.

Holt-Giménez, E. (2002). Measuring farmer’s agroecological resistance after Hurricane Mitch in Nicaragua: A case study in participatory, sustainable land management impact monitoring. Agriculture, Ecosystems and Environment, 93. Retrieved from http://www.sciencedirect.com/science/article/pii/S0167880902000063

INEGI (Instituto Nacional de Estadística y Geografía). (2015). Marco geoestadístico nacional. Disponible en http://gaia.inegi.org.mx/mdm6/

IPCC (Intergovernmental Panel on Climate Change). (2014). Mitigation of climate change. Retrieved from http://www.ipcc.ch/pdf/assessment-report/ar5/wg3/ipcc_wg3_ar5_chapter11.pdf

Lambe, T. W. y Whittman, R. V. (2004). Mecánica de suelos. México: Limusa

Moeys, J. (2015). The soil texture wizard: R functions forplotting, classifying, transforming and exploring soil texture data. Retrieved from http://rforge.rproject.org/scm/viewvc.php/ ${ }^{*}$ checkout*/pkg/soiltexture/inst/doc/ soiltexture_vignette.pdf ?revision $=15 \&$ root $=$ soiltexture\&pathrev $=20$

Nogués, D. y Pérez, F. (2001). Cartografía y análisis espacial del paisaje vegetal de la montaña Riojana y su papel como herramienta de gestión. Zubia. Disponible en https://dialnet.unirioja.es/servlet/articulo? codigo $=664549$

Peck, R. B., Hanson, W. E. y Thornburn, T. H. (2008). Ingeniería de cimentaciones. México: Limusa

Porta, J., López-Acevedo, M. y Poch, R. M. (2014). Edafología: uso y protección de suelos (tercera edición). España: Mundi-Prensa

Purnamita, D., \& Morton, J. (2014). Climate change impacts, adaptation and vulnerability. Retrieved from http://www.ipcc.ch/report/ar5/wg2/

Ramírez, G., Ruiz-Corral, J. A., Pérez, C., Villavicencio, R., Mena, S. y Durán, N. (2014). Impactos del cambio climático en la distribución geográfica de de Gossipium hirsutum L. en México. Revista mexicana de ciencias agricolas, 10. Disponible en http://cienciasagricolas.inifap.gob.mx/editorial/index.php/ Agricolas/article/view/3876 
Rodriguez, G. (2008). Relation between vegetation diversity and soil functional diversity in native mixed-oak forests. Soil Boilogy and Biochemistry, 40. Retrieved from http://www.sciencedirect.com/science/article/ pii/S0038071707001745

Rubio, M. J. y Varas, J. (2004). El análisis de la realidad en la intervención social. Métodos y técnicas de investigación. España: CCS.

UNFCCC (United Nation Framework Convention on Climate Change). (2014). Retrieved from http://unfccc. int/2860.php.

USDA (United States Department of Agriculture). (2016). Soil texture calculator. Disponible en http://www. nrcs.usda.gov/wps/portal/nrcs/detail/soils/survey/?cid=nrcs142p2_054167

Valdez, J. R., González, M. de J. y De los Santos, H. M. (2006). Estimación de cobertura arbórea mediante imágenes satelitales multiesprectales de alta resolución. Agrociencia, 40(3). Disponible en https://dialnet. unirioja.es/servlet/articulo?codigo $=1976305$

Whiting, D., Wilson, C., \& Card, A. (2002). Estimating soil texture: Sandy, loamy, or clayey? Colorado State University cooperative extension. Retrieved from http://culter.colorado.edu/ kittel/SoilChar(\%26RibbonTest)_handout.pdf

CC BY-NC-ND 